\title{
OSTEOARTHRITIS
}

\section{Genetically modified ADSCs as cell therapy for OA?}

Genetic modification of adipose-derived stromal cells (ADSCs) could form the basis of a new therapeutic approach for osteoarthritis (OA), according to a paper published in Arthritis \& Rheumatology. As the lead author of the paper Aline Bozec explains, "The study was part of the ADIPOA European Research project to demonstrate the proof-of-concept of ADSCs in OA treatment and to try to understand the mechanisms involved."

ADSCs, like mesenchymal stem cells (MSCs), can self-renew and differentiate into chondrocytes, osteoblasts and adipocytes; however, ADSCs are more prevalent than MSCs and can be isolated easily by liposuction, making them an attractive potential cell therapy for OA. Previous research showed that intraarticular injection of ADSCs reduces OA disease severity in mice and rabbits. Could genetic modification of these cells improve their therapeutic potential?

"Mice over-expressing the transcription factor Fra-1 (Fra-1tg mice) develop progressive osteosclerosis due to cell-autonomous accelerated bone formation by the osteoblast," says Bozec, suggesting that Fra-1 could be a good gene to target in ADSCs to improve the chondroprotective effects of these cells.

\section{Cartilage destruction and chondrocyte death were reduced in mice treated with Fra-1tg ADSCs... 77}

Fra-1tg ADSCs or wild-type ADSCs were isolated from the inguinal fat pads of 8-week-old mice and injected into knee joints of C57BL/ 6 mice 7 days after induction of collagenase-induced osteoarthritis. Cartilage destruction and chondrocyte death were reduced in mice treated with Fra-1tg ADSCs compared with those that received wild-type ADSCs. Fra-1tg ADSCs were found to comprise nearly twice as many adipogenic progenitors than wild-type ADSCs, but this was not the reason for the improved chondroprotection provided by these cells, as enriching the numbers of progenitors in wild-type ADSCs had little effect.

The authors analyzed the molecular characteristics of the Fra-1tg ADSCs. These cells expressed decreased levels of the proinflammatory mediators IL- 6 and pentraxin, and increased levels of the extracellular matrix proteins periostin and spondin-1. "We speculate that the improved protective effect of Fra-1tg ADSCs on cartilage integrity is mediated by a comprehensive change in gene expression pattern based on downregulation of inflammation genes and fostering expression of extracellular matrix genes," concludes Bozec. "However, further studies are necessary to define the factor essential for this protective effect."

Jenny Buckland

Original article Schwabe, K. et al. Adipose-derived stromal cells expressing Fra-1 inhibit osteoarthritis. Arthritis Rheumatol. doi:10.1002/art.39425 